\title{
Ketamine: An Introduction for the Pain and Palliative Medicine Physician
}

Tomasz Okon, MD

\begin{tabular}{|c|c|}
\hline $\begin{array}{r}\text { From: Department of Palliative } \\
\text { Medicine, Marshfield Clinic, Marshfield, } \\
\text { WI. } \\
\text { Dr. Okon is Clinical Assistant Professor, } \\
\text { University of Wisconsin Madison; } \\
\text { Fellowship Director-Palliative Medicine } \\
\text { Fellowship, Marshfield Clinic, Marshfield, } \\
\text { WI. } \\
\text { Address Correspondence: } \\
\text { Tomasz Okon, MD } \\
1000 \text { North Oak Avenue } \\
\text { Marshfield, WI } 54449 \\
\text { Email: okon.tomasz@marshfieldclinic.org }\end{array}$ & $\begin{array}{l}\text { A history of an escalating chronic intractable pain in a patient with cryoglob- } \\
\text { ulinemia, vasculitis, and severe cutaneous ulcerations is presented. A strategy } \\
\text { of progressive, multi-agent, N-methyl-D-aspartate-receptor (NMDA-R) blockade } \\
\text { that resulted in adequate pain control and a three-fold reduction in opioid con- } \\
\text { sumption is described. Diagnostic process of neuropathic pain and the role of } \\
\text { NMDA-R in the development of hyperalgesia are briefly reviewed. Thereafter, ex- } \\
\text { isting clinical literature describing the use of Ketamine, a major NMDA-R antago- } \\
\text { nist for management of malignant pain, is reviewed. Lastly, evidence-based orig- } \\
\text { inal protocol for intravenous adjuvant Ketamine analgesia for severe cancer pain } \\
\text { is presented. }\end{array}$ \\
\hline $\begin{array}{r}\text { Funding: None. } \\
\text { Conflict of Interest: None. } \\
\text { Presented in part at the 8th Annual } \\
\text { Josefina Magno Coference, Falls Church, } \\
\text { VA, October } 2006 .\end{array}$ & $\begin{array}{l}\text { Key Words: Cancer, Ketamine, pain } \\
\text { Pain Physician 2007; 10:493-500 }\end{array}$ \\
\hline $\begin{array}{r}\text { Manuscript received on: 04/16/2007 } \\
\text { Revisions accepted: 04/20/2007 } \\
\text { Accepted for publication on: 04/24/2007 }\end{array}$ & \\
\hline $\begin{array}{r}\text { Free Full manuscript: } \\
\text { www.painphysicianjournal.com }\end{array}$ & \\
\hline
\end{tabular}

Dersistent noxious stimuli lead to the development of a phenomenon known as central sensitization whereby stimuli of stable intensity result in progressively higher pain intensity. $\mathrm{N}$-methyl-D-aspartate-receptor (NMDA-R) contributes to the process of sensitization by generating pain hypersensitivity upon a variety of post-translational modifications (1). Conversely, NMDA-R antagonists, including Ketamine and methadone, are well known to attenuate central sensitization and palliate neuropathic pain.

We describe a successful treatment of intractable pain with neuropathic features by utilizing a combined, 2 agent strategy of escalating NMDA-R block- ade and review existing literature and clinical protocols for adjuvant Ketamine analgesia.

\section{Case Description}

\section{Phase I}

This 41-year-old woman presented to the palliative medicine service of Marshfield Clinic, Marshfield, Wisconsin in February of 2005 in consultation from the hospitalist service to determine whether her analgesic regimen was safe and effective.

The patient presented with a history of hepatitis $C$, cirrhosis, and chronic nonmalignant pain described as discomfort with nociceptive features in her lower 
extremities bilaterally. The pain was achy without neuropathic features. It worsened with walking and with periodic leg swelling. Intensity at worst was about 7 out of 10. At the time of the interview, she rated the pain as 3 out of 10 . Her goal of relief, set in the range of average pain of 3 to 4 out of 10, was determined by an acceptable level of physical functioning that the patient was able to achieve with the residual (3-4/10) discomfort.

She also had a mild abdominal discomfort not affecting her level of functioning. With a daily dose of $20 \mathrm{mg}$ of oxycodone SR (10 mg twice daily) and a breakthrough dose of oxycodone IR $5 \mathrm{mg}$ every 6 to 8 hours prn, she was comfortable and free of side effects. Calculated daily oral morphine equivalent dose (MED) was in the range of 45 to $60 \mathrm{mg}$. The palliative medicine consultant recommended continuation of the analgesic regimen.

Shortly thereafter, the patient became eligible for inclusion on the liver transplant list. A transplant team requested that all of her pain prescriptions be issued exclusively by an outside pain and addiction clinic. Henceforth, she was lost to follow-up by the primary pain and palliative medicine team for several months.

\section{Phase II}

In February 2006, a nephrologist, who supervised this patient's scheduled plasmapheresis for vasculitis attributable to cryoglobulinemia that was diagnosed in the interim period, requested direct admission to the palliative care unit for management of very poorly controlled pain. The pain was intermittent and extending versus radiating from the bottom of her left foot up to the knee. Baseline discomfort was rated as 4-5/10; at worst she rated the pain at 7-8/10. She described it as, "if somebody had stuck a knife in me and turned it." Furthermore, she reported a numbing and burning component, primarily in her left foot. On physical examination that included formal microfilament sensory testing, she had distinct deficits in her sensory function in the lower extremities, left more than right. Multiple open ulcerations on the lower extremities were also noted, without purulence. Her analgesic regimen included fentanyl TD $50 \mu \mathrm{g} /$ hour and oral oxycodone (5$10 \mathrm{mg}$ dose) prn for a daily dose total of $120 \mathrm{mg}$, hence MED in the range of 400-500 mg. Adjuvant analgesia consisted of gabapentin $900 \mathrm{mg}$ and prednisone 20 mg/day. Non-steroidal anti-inflammatory drugs (NSAID) and acetaminophen were not administered due to the underlying renal and hepatic conditions.
Since the pain was worse with weight bearing, a bone scan was ordered which showed no pathologic involvement. The opioid regimen was rotated to methadone according to the British conversion protocol (2). On discharge she was receiving $35 \mathrm{mg}$ bid and $10 \mathrm{mg}$ every 3 hours prn for pain. Vigilant follow-up was scheduled and the patient returned 2 weeks postdischarge reporting somnolence. Methadone was adjusted to $20 \mathrm{mg}$ bid, along with $10 \mathrm{mg}$ of oxycodone every 3 hours prn for a total MED in the $500-600 \mathrm{mg}$ range.

To summarize, her daily oral MED that started at about 45 to $60 \mathrm{mg}$ was increased 10 -fold during the 10 month period of disease progression. The analgesic dose escalation did not result in adequate pain relief. Thereafter, rotation to methadone afforded adequate pain relief, likely attributable to the utilization of the mixed, $\mu$-receptor agonist and NMDA-R antagonist (methadone). The rotation itself did not result in escalation in the total MED.

\section{Phase III}

The patient was admitted a few months later with a 3-4 day history of progressive, intractable, right leg pain and mental status change. Initially, the pain exacerbation was attributed to cellulitis and further worsening of leg ulcerations.

Physical examination of the lower extremities revealed not only numerous deep, small (about 4-6 mm in diameter and 3-4 $\mathrm{mm}$ in depth) ulcerations, but also an element of erythema consistent with cellulitis. She was treated with antibiotics, while the previous regimen of methadone remained unchanged.

With the aggressive medical treatment, the pain improved as did the signs of acute infection, but she still reported a residual discomfort described in subjective terms consistent with complex nociceptive-neuropathic pain, which remained a primary source of her personal sense of discomfort.

Due to persistent pain, adjuvant treatment with oral Ketamine $20 \mathrm{mg}$ tid was then started. The pain improved further to the 5-6/10 range (at personal goal of pain relief). Furthermore, reduction in the methadone dose from 20 to $15 \mathrm{mg}$ bid was possible due to achievement of adequate analgesia. Ritalin 10 mg every morning was added with an excellent level of alertness and cognition resulting from this adjuvant approach.

At discharge, her opioid regimen consisted of methadone $15 \mathrm{mg}$ bid and oxycodone $10 \mathrm{mg}$ every 3 hours prn breakthrough pain. Adjuvant analgesia con- 
sisted of Gabapentin 800 mg tid, Ketamine 20 mg tid, prednisone $20 \mathrm{mg} / \mathrm{day}$, and Ritalin. Thus, her MED was decreased from the previous 600 to about $450 \mathrm{mg}$.

Two months later the plasmapheresis supervising nephrologist reported that the patient had terrible pain. On interview, she revealed that she had stopped Ketamine due to somnolence. She was asked to restart the Ketamine and was followed up with a clinic visit within a week. At that time Ketamine was further adjusted to $15 \mathrm{mg}$ tid. Other medications were unchanged.

Within the subsequent 4 months, methadone was decreased to $7.5 \mathrm{mg}$ twice daily. Oxycodone was continued at $10 \mathrm{mg}$ every 3 hours prn and Ketamine 15 $\mathrm{mg}$ bid in addition to Gabapentin and Ritalin. By the time of the last available data set from late September 2006, she reported "no pain" or "mild" intensity pain, with MED in the $200 \mathrm{mg}$ range, which amounted to a dramatic decrease in her opioid consumption with excellent pain control. At the time of submission, she was no longer contemplating or eligible for transplantation.

\section{Neuropathic Pain}

The type of discomfort the patient suffered initially was primarily nociceptive pain in the lower extremities, which became chronic and intractable. Because of the severity and intractability of the pain, it facilitated a so-called "central sensitization" phenomenon, whereby a prolonged excitation of the dorsal horn causes over-activation (and possibly, over expression) of the NMDA-R.

Central sensitization may be conceptualized as a signal amplifier that, over time, is inserted into the pain conducting circuitry. Thereby, previously innocuous stimuli become exaggerated and perceived as painful. Clinically, central sensitization can manifest in a variety of ways. Two that are classic and frequently used in experimental studies are secondary hyperalgesia and wind-up. Secondary hyperalgesia amounts to perception of pain outside the area initially injured; e.g., if one slightly burns the tip of a finger and eventually the entire finger pains, the extension of pain is due to secondary hyperalgesia. This is one clinical manifestation of central sensitization. The wind-up phenomenon consists of repeated transmission of nociceptive stimuli (clinically present with protracted pain) resulting in summation of the stimuli. This leads to exaggeration of pain (hyperalgesia) on repeated exposure to mildly nociceptive, or otherwise innocu- ous stimuli. Both phenomena depend on activation of the NMDA-R.

With regard to the wind-up phenomenon, intense or sustained noxious stimuli generate a high frequency signal that results in the co-release of excitatory amino acid (EAA) and produce slow, lasting potentials. This leads to temporal summation of nociceptive transmission. The resulting cumulative depolarization is amplified by the additional NMDA-R current, which is triggered by removal of the magnesium-dependent blockade of NMDA-R. Depolarization also activates voltage-gated calcium currents triggering plateau potentials mediated by calcium-activated ion channels. A wind-up of action potential discharge ensues (3).

NMDA-R activation is also involved in the amplification of physiologic $\alpha$-amino-3-hydroxy-5-methylisoxazole-4-propionic acid-receptor (AMPA-R) responses at synapses on dorsal horn neurons. This very complex yet reversible process of modulation of receptor response is initiated by a signaling cascade involving enhancement of the NMDA-R function by the tyrosine kinase, raised intracellular calcium, activation of kinase II and protein kinase C, and enhanced AMPA channel conductance and/or cell-surface expression.

Our patient's objective pain history alone suggests that she may have suffered from neuropathic pain due to the NMDA-R mediated central sensitization triggered by her intractable chronic pain. Furthermore, her symptoms were spontaneously and explicitly described as "burning" and "numbness," a second line of evidence for the diagnosis of neuropathic pain.

According to a well validated instrument, a neuropathic pain questionnaire (4), the 3 most discriminating descriptors of neuropathic pain are tingling, increased pain with touch, or numbing (mnemonic: TIN-gling). In addition, one may elicit a history of burning, shooting pain (but not electric pain), or freezing pain.

In the Leeds assessment of neuropathic symptoms and signs (LANSS) scale (5), the additional inclusive diagnostic features are subjective descriptors such as "prickling," perceived alterations in the sense of temperature or skin color, and objective findings of either allodynia, hyperalgesia, or hypoalgesia.

Allodynia is pain elicited by a non-noxious stimulus, while hyperalgesia is an exaggerated pain response to a mildly noxious (mechanical or thermal) stimulus. Our patient did have a sensory loss consistent with hypoalgesia. However, later she developed allodynia, the third objective finding consistent with the neuropathic component of her discomfort. 
We used methadone at the time of the initial suspicion of neuropathic pain because we discerned 3 lines of evidence in support of such a diagnosis: objective elements of medical history, subjective symptoms, and objective signs on examination. Once a neuropathic pain is diagnosed, one may empirically use medications proven to be effective in one neuropathic condition for treatment of other neuropathic conditions (6). This approach amounts to an educated, informed error and trial, or a clinical empiricism strategy. Several recent landmark studies and excellent review articles can be found on this topic (7-9).

Most importantly, when the pain further escalated, we introduced another adjuvant agent, Ketamine, with excellent overall results as demonstrated by the eventual reduction of MED from about $700 \mathrm{mg} /$ day at the peak opioid consumption to about $200 \mathrm{mg} / \mathrm{day}$ at the time of last discharge from the hospital. In both steps, we conceptualized the operating therapeutic rationale as a stepwise, progressive antagonism of NMDA-R.

\section{Ketamine}

Ketamine was developed as an anesthetic. It has weak primary analgesic properties (10) when used by itself in smaller doses. In other circumstances and uses, however, it exerts strong adjuvant analgesic properties by inhibiting the binding of glutamate to the NMDA$\mathrm{R}$, which is probably its most critical mechanism of action. Ketamine exhibits a "state-dependency" in that it modulates aberrant behavioral and neuronal responses induced by neuropathic injury (inhibits transmission/amplification of painful stimuli), yet has little effect on normal neuronal function. Its interaction with NMDA-R is mediated via its binding to the phencyclidine (PCP) site with much higher affinity than to other receptors (or voltage-gated channels). At subanesthetic doses, NMDA-R is the primary binding site for Ketamine and hence likely the one responsible for the clinically observed analgesia.

The primary endogenous neurotransmitter active at NMDA-R is glutamate, the main EAA. It is probable that the action of the excitatory neurotransmitter glutamate on NMDA-R facilitates sensitization of spinal neurons and progression of nociceptive symptoms, including endogenous and opioid induced hyperalgesia (11). Highly permeable to calcium, NMDA-R mediates intracellular elevation of calcium, leading to a cascade of excitatory events. The sequence of these intracellular signaling events is complex. However, they seem to result in the activation of protein kinase $\mathrm{C}$ and el- evation of levels of nitrous oxide, which in turn, leads to enhanced release of other EAAs. This action of NMDA-R is blocked by magnesium in a voltage-dependent fashion. Conversely then, once the synaptic membrane has been depolarized (for instance by a prolonged pain stimulus), the magnesium block is removed and excitatory sequence results. NMDA-R antagonists such as Ketamine, by binding to the PCP site, block the NMDA-R, decreasing the pro-nociceptive neurotransmission.

Furthermore, activation of the NMDA-R has been shown to modulate opioid receptor mediated functions, such as the paradoxical development of opioid tolerance on the one hand and hypersensitivity on the other. Homosynaptic location of NMDA-R and $\mu$ receptors has also been confirmed, providing an anatomic substrate of the receptors interaction model (12). Conversely, administration of NMDA-R antagonists have been shown to reduce neuro-excitation caused by high-dose morphine (13) and remifentanil in humans (14). Most importantly, there is growing evidence that NMDA-R antagonists, such as Ketamine, palliate spontaneous, neuropathic pain. However, there may be other mechanisms of Ketamine's action.

Ketamine is active at norepinephrine, serotonin, and muscarinic cholinergic receptors (15). Indeed, some studies suggest that analgesic effects of Ketamine are unrelated to its binding to the NMDA-R, but rather are due to the activation of monoaminergic descending inhibitory pathways. Importantly, as a catecholamine reuptake inhibitor, Ketamine elevates circulating levels of epinephrine and norepinephrine. This mechanism proved on several occasions to be significant in the author's clinical experience, in that it led to instances of supraventricular tachycardias, necessitating drug discontinuation. Lastly, while the beneficial effects of Ketamine on inflammatory pain may be NMDA-R mediated, its effect on acute, non-inflammatory pain could involve a descending inhibitory system (16). Lastly, Ketamine's action on opioid receptors remains controversial, given the inconsistent results of naloxone on reversal of Ketamine effects.

In regard to pharmacokinetics, Ketamine has a short half-life of about 3 hours. Thus, with scheduled administration, a steady state is achieved in 12-15 hours." When administered orally, $80 \%$ of the Ketamine dose undergoes hepatic metabolism via the cytochrome P450 system to norKetamine. Hence, bioavailability is about $20 \%$. NorKetamine, which is ex- 
creted in urine, is thought to have about $30 \%$ of the analgesic potency of the parent drug. A dose reduction in patients with hepatic impairment is advised due to the prolonged duration of action. In renal failure, dose increases may be considered.

Due to its small size and lipophilic character, Ketamine crosses the blood brain barrier, leading to the onset of action within 1-3 minutes. Timing to maximum pain relief remains a controversial issue, since it depends on the mechanism of the pain. In the Mercadante et al series (17), maximum pain relief after a single intravenous dose occurred between 30 and 60 minutes after the infusion. However, there are practitioners who use it on an as needed basis only, and report adequate pain control as late as 5 days after a single infusion (18).

In regard to the side effect profile, adverse cardiovascular effects are frequent and include increased blood pressure and tachyarrhythmias. Neurologic adverse effects include increased intracranial pressure, and increased muscle tone. Cognitive complications are frequent and manifest primarily in a psychomimetic fashion: alterations in body image and mood, floating sensations, vivid dreams, hallucinations, delirium, and drowsiness. The incidence of psychotomimetic adverse effects is greatest in patients with a history of psychosis or personality disorders. Only occasionally does respiratory depression occur. Chronic Ketamine use may result in lasting memory and cognitive dysfunction (19). Only occasionally does respiratory depression occur, primarily with concurrent opioid exposure. Increased lacrimation and salivation have been reported with anesthetic doses only.

\section{Clinical Protocols for Ketamine Use}

There is no agreement on a single, uniform best Ketamine protocol or dose. Instead, various local, idiosyncratic approaches are used. One typical, intermittent dosing schedule is $0.25-0.50(0.60) \mathrm{mg} / \mathrm{kg}$ tid. While some practitioners use the intravenous route (17), others prefer oral delivery (20). Most start at that same dose $(0.25-0.50 \mathrm{mg} / \mathrm{kg})$, however. Similarly, initial doses of $0.8 \mathrm{mg} / \mathrm{kg} /$ day in divided doses by oral or subcutaneous route (which amounts to about $2 \mathrm{mg} /$ hour for a $60 \mathrm{~kg}$ [130 lb] person) (21) have been described. Others utilize continuous, non-weight based infusion starting at $100 \mathrm{mg} / \mathrm{day}$ ( 4 mg/hour) (22). Alternatively, Prommer (21) described a protocol initiated with an intravenous loading dose of $0.5 \mathrm{mg} / \mathrm{kg}$ over 30 minutes, followed by continuous, non-weight based infusion starting at $2 \mathrm{mg} /$ hour titrated to effect. Fine (24) suggests the use of escalating bolus starting at $0.1 \mathrm{mg} / \mathrm{kg}$ intravenously or $0.5 \mathrm{mg} / \mathrm{kg}$ subcutaneous, and doubling it every 15 minutes for the intravenous route or 30-45 minutes for the subcutaneous route until the pain improves. Thereafter, continuous infusion commences at the effective dose determined by the initial, dose-finding trial. Corell et al (25) described utilization of the starting dose of $10 \mathrm{mg} / \mathrm{hour}$ followed by upwards titration and continuation for a median infusion duration of 4 days and a median final infusion rate of $20 \mathrm{mg} / \mathrm{hour}$ for patients with regional pain syndrome.

Ketamine titration for incomplete pain relief has been described in equally varied terms. Jackson et al (26) described a protocol whereby the dose is increased every 24 hours from $100 \mathrm{mg} /$ day ( $4 \mathrm{mg} / \mathrm{hour}$ ) to $300 \mathrm{mg} / \mathrm{day}(\sim 12 \mathrm{mg} / \mathrm{hour})$, and then to $500 \mathrm{mg} /$ day ( $20 \mathrm{mg} / \mathrm{hour}$ ) on the third day. Treatment is discontinued after 5 days, if no analgesic effect is reached with $500 \mathrm{mg} /$ day infusion. In a slight modification of that protocol, Good et al (22) titrated the dose up to $700 \mathrm{mg} /$ day with no reported unacceptable adverse effects. Corell et al (25) described daily $25 \%-50 \%$ upward titration to analgesic effect versus the development of side effects.

Parenteral to enteral transition is anecdotally said to allow for up to $50 \%-60 \%$ of dose reduction (27). This cannot be considered a standard of practice in the context of other studies (20) and what we presently know about pharmacokinetics and bioavailability of Ketamine and its metabolites.

After Ketamine is started, reduction of opioid dose within the first 12 days of infusion must be attempted unless pain remains uncontrolled. Hence, assessments of pain, vital signs, adverse effects, and the comprehensive clinical monitoring are mandatory, at least bid on the outpatient basis, and more frequently with inpatients.

One should strongly consider the routine use of a small dose of benzodiazepine or neuroleptic while initiating treatment to minimize the psychotomimetic side effects. Given the frequent adverse cardiac effects of Ketamine, adding butyrophenones could potentially compound these effects, and therefore a benzodiazepine is better used for this purpose. Intravenous or subcutaneous lorazepam $1 \mathrm{mg}$ at the outset of infusion and then every 3 hours up to 3 doses/day prn dysphoria, dissociation, or restlessness is probably a safe and effective dose (Table 1). 
Table 1. Marshfield Inpatient Analgesic Ketamine Protocol

Discuss side effects very thoroughly with the patient prior to orders to prepare (but not to needlessly alarm) patient and family for the likely psychotomimetic effects. Reassure frequently if needed during the infusion.

Start at $0.5 \mathrm{mg} / \mathrm{kg}$ intravenous over 6 (4) hours (average dose $30 \mathrm{mg}$, i.e. $5 \mathrm{mg} /$ hour x 6 hours).

- Note: this is the most effective dose from Mercadante's study extended over a safer infusion time (4-6 hours vs 30 minutes), yet shortened from the Australian protocol calling for a 24-hour trial.

Order $1 \mathrm{mg}$ of lorazepam at the beginning of infusion and every 3 hours $\mathrm{x} 2$ subsequent doses prn psychotomimetic side effects. Alternatively, may give $2 \mathrm{mg}$ of Haloperidol. Order glycopyrrolate $0.2-0.3 \mathrm{mg}$ subcutaneous every 6 hours prn excessive salivation or lacrimation.

Check psychotomimetic side effects and vitals every 1 hour x 3, pain intensity every 2-3 hours.

- Stop infusion if $\mathrm{P}>110$, systolic blood pressure increased by more than $25 \%$ of baseline, sustained $\mathrm{RR}<7$, agitation or severe, intolerable psychotomimetic side effects.

If pain improved by $50 \%$ or more during the initial infusion continue with intravenous infusion at a total dose of $1.5 \mathrm{mg} / \mathrm{kg} / \mathrm{day}$ for 48 (72) hours, than convert 1:1 to subcutaneous tid.

- Note that this dose for an average weight person yields a rate close to the Australian protocols ( $\pm 100 \mathrm{mg} /$ day $)(22,26)$. Oral conversion is a matter of dispute. I tend not to decrease the dose on the parenteral to enteral conversion.

If pain not improved and no severe side effects, start $2 \mathrm{mg} / \mathrm{kg}$ infusion over the next 12 hours (effectively doubling the initial dose).

- Note, in the latter phases, unlike some described protocols $(23,24)$, I wait the entire five elimination half-times before the next round of titration.

If pain recurs, titrate upwards by $50 \%-100 \%$ every 24 hours.

\section{Outcomes Reported with Ketamine Use for Cancer Pain}

Mercadante et al (17) compared intravenous infusions of Ketamine $(0.25$ and $0.5 \mathrm{mg} / \mathrm{kg})$ with placebo in a double-blind, crossover study of 10 cancer patients with neuropathic pain. All patients were also receiving morphine (MED 60-300) and were given the two different doses of Ketamine and placebo. Single daily infusions were used over 30 minutes, each at least 2 days apart with at least 3 repetitions. Mean pain intensities (as measured on a 0-10 scale) were reduced from 6.6 to 1.4 (0.25 mg/kg dose), and from 5.9 to 0.2 after administration $0.5 \mathrm{mg} / \mathrm{kg}$. This compares with essentially no change (mean 6.5) after placebo administration. Pain relief from Ketamine $0.25 \mathrm{mg} / \mathrm{kg}$ and 0.5 $\mathrm{mg} / \mathrm{kg}$ was sustained over the 180-minute observation period. There was a gradual increase in pain intensity noted (3.8/10 and $1.8 / 10$ at 180 minutes for the 0.25 and $0.5 \mathrm{mg} / \mathrm{kg}$ doses, respectively). Four of 10 patients experienced psychotomimetic effects with Ketamine, reduced with intravenous diazepam administration.

Kannan et al (20) used oral Ketamine $(0.5 \mathrm{mg} / \mathrm{kg}$ 3 times daily) in combination with other analgesics to treat 9 patients with neuropathic cancer pain. All patients were taking high doses of morphine, amitriptyline, and sodium valproate. All reported initial pain rat- ings greater than 6 on a $0-10$ scale when Ketamine was started. Seven of the 9 patients experienced a decrease of more than 3 points in the average pain score.

In a recent study, Good et al (22) demonstrated that the Ketamine-based, triple-agent approach is safe and effective in an inpatient palliative care population during episodes of poorly controlled acute or chronic cancer pain. Inclusion criteria for this 6 month long prospective audit were: 1) unstable pain control with moderate to severe pain requiring hospital admission, 2) poor response to prior therapy - on admission, the median dose of parenteral morphine equivalent was $66 \mathrm{mg} / 24$ hours with a range of $12.5-450 \mathrm{mg} / 24$ hours, 3) high risk (for poor control) pain mechanisms and syndromes, especially incident and/or neuropathic pain. All patients were treated with a combination of Ketamine, an opioid, and an anti-inflammatory agent (either dexamethasone or one of the following: ketorolac, naproxen, or parecoxib). The starting dose of Ketamine was $100 \mathrm{mg} /$ day and the final median dose of Ketamine was $200 \mathrm{mg} / 24$ hours (just short of $10 \mathrm{mg} / \mathrm{hour}$ ) with a range of 100-700 mg/day. The median number of days of Ketamine use was 5 with a range of 3-17 days. Ketamine dosage was adjusted according to responses during the observation period. Respond- 
ers were defined as:

1. Achieve a verbal rating scale (VRS) score of 0 using a graded scale where $10=$ worst imaginable pain and $0=$ no pain; or

2. Have a $50 \%$ or greater reduction in mean VRS.

In addition, one of the following 2 surrogate criteria had to be fulfilled:

- Fifty percent or greater reduction in the 24 hour opioid dose or a $50 \%$ or greater reduction in the number of opioid breakthrough doses, or

- Improvement in mobility or function.

Of the total 18 patients who were studied, 12 were classified as responders for an excellent response rate of $66 \%$.

In an earlier study, Jackson et al (26) reported Ketamine use in refractory cancer pain. In this prospective, multicenter, unblinded, open-label audit, 39 patients (with a total of 43 pain clusters) received a short duration (3 to 5 days) Ketamine infusion. The initial dose of $100 \mathrm{mg} / 24$ hours was escalated if required to $300 \mathrm{mg} / 24$ hours and then to a maximum dose of $500 \mathrm{mg} / 24$ hours. Fifteen of 17 somatic and $14 / 23$ neuropathic pains responded with definition of response very close to that utilized in the Good et al study (22).

A systematic review published before completion of Good's study (28) demonstrated absence of high quality studies and determined that the evidence available had not been sufficient to make a recommendation for routine use of any specific dosing protocol of Ketamine for cancer pain. This conclusion was subsequently disputed by a variety of responders to Bell et al paper (28).

\section{Other NMDA-R Antagonists}

A summary of published reports on other NMDA$\mathrm{R}$ antagonists, including dextrometorphan, amantidine, memantidine, PCP, and magnesium is available elsewhere (29).

\section{Discussion}

Dr. Okon (replying to a question of concurrent methadone and ketamine use):

In this relatively unusual case - and it is not a frequent case that I venture to use methadone and Ketamine at the same time - the rationale was the following: methadone has a fixed ratio between the agonistic action on opioid receptor and the antagonistic action on NMDA-R. Since the patient experienced long, intractable pain, she must have had a tremendous windup and central sensitiza- tion, while the NMDA-Rs were proportionally activated. Hence, an additional NMDA-R blockade (beyond what might have been achieved with methadone alone) seemed like a responsible and reasonable plan.

A case was made for escalating the NMDA-R blockade with an additional NMDA-R antagonist. I definitely, am not willing to say that this approach should be perceived as a recommendation, or a common practice. In fact, I more frequently use a pure opioid receptor agonist and Ketamine than methadone along with Ketamine. In this case, however, I would have had to withdraw the patient from methadone, try to reconvert the opioid, most likely to fentanyl given her renal co-morbidities, and finally add Ketamine. The clinical urgency of controlling pain while not prolonging the hospitalization beyond what was necessary led me to the other, just presented course of action.

In regard to dextramathorphan, I do not use it frequently because earlier in my career it seemed that the prevailing sentiment was that dextramathorphan had a fairly low potency at NMDA-R as compared to other effects. Given the low therapeutic ratio, I just do not use dextramathorphan much.

\section{Dr. Giordano speaking from the audience:}

The use of more than one NMDA-R antagonist makes sense with regard to the clinical pharmacology and some of the neuroscience of what is going on with neuropathic pain. Very recently Cliff Wolfe has had a very nice schemata about trying to diagnosis neuropathic pain and then developing that as proof of concept. If we go back and if we take a look at some of the evidence of what is going on with regard to neuropathology of that pain.

In part, what happens is that there are really 3 distinct NMDA type receptors that are capable of sensitization of regulation as you have alluded to. But the NMDA-R which is the gated receptor from (inaudible) the magnesium gated, the metabotropic glutamate receptors which are two subtypes and the ionotropic glutamate receptor. All 3 are capable of some form of upregulation or modification and they are differentially sensitive to different pharmacologic agents. The rationale of using more than one NMDA or putative NMDA-R antagonist is that there really is no way to determine apriori what particular upregulated 
subpopulation of NMDA-Rs a given patient may have based on the genomics of that as a special event.

There is nothing sentinel that can tell the clinician whether to use drug $x$ versus drug $y$ but the use of a combinatory regimen of NMDA-R an- tagonist would then differentially hit all of those subpopulations on an empiric basis so I applaud you for doing that. That is the take home message there. I do not think there is any way, at this point, to be able to determine $x$ is upregulated and $y$ is not.

\section{REFERENCES}

1. Brenner GJ, Ji RR, Shaffer S, Woolf CJ. Peripheral noxious stimulation induces phosphorylation of the NMDA receptor NR1 subunit at the PKC-dependent site, serine-896, in spinal cord dorsal horn neurons. Eur J Neurosci 2004; 20:375384 .

2. Morley JS, Watt JW, Wells JC, Miles JB, Finnegan MJ, Leng G. Methadone in pain uncontrolled by morphine. Lancet 1993; 342:1243.

3. Woolf CJ, Thompson SW. The induction and maintenance of central sensitization is dependent on N-methyl-D-aspartic acid receptor activation; implications for the treatment of post-injury pain hypersensitivity states. Pain 1991; 44:293-299.

4. Krause SJ, Backonja MM. Development of a neuropathic pain questionnaire. Clin J Pain 2003; 19:306-314.

5. Bennett M. The LANSS Pain Scale: the Leeds assessment of neuropathic symptoms and signs. Pain 2001; 92:147-157.

6. Hansson PT, Dickenson AH. Pharmacological treatment of peripheral neuropathic pain conditions based on shared commonalities despite multiple etiologies. Pain 2005; 113:251-254.

7. Mendell JR, Sahenk Z. Clinical practice. Painful sensory neuropathy. $N$ Engl J Med 2003; 348:1243-1255.

8. Rowbotham MC, Twilling L, Davies PS, Reisner L, Taylor K, Mohr D. Oral opioid therapy for chronic peripheral and central neuropathic pain. $N$ Engl J Med 2003; 348:1223-1232.

9. Gilron I, Bailey JM, Tu D, Holden RR, Weaver DF, Houlden RL. Morphine, gabapentin, or their combination for neuropathic pain. N Engl J Med 2005; 352:1324-1334.

10. Owen H, Reekie RM, Clements JA, Watson R, Nimmo WS. Analgesia from morphine and Ketamine. A comparison of infusions of morphine and Ketamine for postoperative analgesia. Anaesthesia 1987; 42:1051-1056.

11. Liu H, Mantyh PW, Basbaum Al. NMDAreceptor regulation of substance $P$ re- lease from primary afferent nociceptors. Nature 1997; 386:721-724.

12. Celerier E, Rivat C, Jun $Y$, Laulin JP, Larcher A, Reynier P, Simonnet G. Longlasting hyperalgesia induced by fentanyl in rats: preventive effect of Ketamine. Anesthesiology 2000; 92:465472.

13. Lufty K, Woodward RM, Keana JF, Weber $E$. Inhibition of clonic seizure-like excitatory effects induced by intrathecal morphine using two NMDA receptor antagonists: MK-801 and ACEA-1011. Eur J Pharmacol 1994; 252:261-266.

14. Angst MS, Koppert W, Pahl I, Clark DJ, Schmelz M. Short-term infusion of the mu-opioid agonist remifentanil in humans causes hyperalgesia during withdrawal. Pain 2003; 106:49-57.

15. Reich DL, Silvay G. Ketamine: an update on the first twenty-five years of clinical experience. Can J Anaesth 1989; 36:186-197.

16. Kawamata $\mathrm{T}$, Omote $\mathrm{K}$, Sonoda $\mathrm{H}$, Kawamata M, Namiki A. Analgesic mechanisms of Ketamine in the presence and absence of peripheral inflammation. Anesthesiology 2000; 93:520528.

17. Mercadante S, Arcuri E, Tirelli W, Casuccio A. Analgesic effect of intravenous Ketamine in cancer patients on morphine therapy: a randomized, controlled, double-blind, crossover, double-dose study. J Pain Symptom Manage 2000; 20:246-252.

18. Mitchell AC, Fallon MT. A single infusion of intravenous Ketamine improves pain relief in patients with critical limb ischaemia: results of a double blind randomised controlled trial. Pain 2002; 97:275-281.

19. Krystal JH, Karper LP, Seibyl JP, Freeman GK, Delaney R, Bremner JD, Heninger GR, Bowers MB Jr, Charney DS. Subanesthetic effects of the noncompetitive NMDA antagonist, ketamine, in humans. Psychotomimetic, perceptual, cognitive, and neuroendocrine responses. Arch Gen Psychiatry 1994:51:199-214
20. Kannan TR, Saxena A, Bhatnagar S, Barry A. Oral Ketamine as an adjuvant to oral morphine for neuropathic pain in cancer patients. J Pain Symptom Manage 2002; 23:60-65.

21. Kotlinska-Lemieszek A, Luczak J. Subanesthetic Ketamine: an essential adjuvant for intractable cancer pain. J Pain Symptom Manage 2004; 28:100102.

22. Good P, Tullio F, Jackson K, Goodchild C, Ashby M. Prospective audit of shortterm concurrent Ketamine, opioid and anti-inflammatory "triple-agent" therapy for episodes of acute on chronic pain. Intern Med J 2005; 35:39-44.

23. Prommer E. Ketamine to control pain. J Palliat Med 2003; 6:443-446.

24. Fine PG. Low-dose Ketamine in the management of opioid nonresponsive terminal cancer pain. J Pain Symptom Manage 1999; 17:296-300.

25. Correll GE, Maleki J, Gracely EJ, Muir JJ, Harbut RE. Subanesthetic Ketamine infusion therapy: a retrospective analysis of a novel therapeutic approach to complex regional pain syndrome. Pain Med 2004; 5:263-275.

26. Jackson K, Ashby M, Martin P, Pisasale M, Brumley D, Hayes B. "Burst" Ketamine for refractory cancer pain: an open-label audit of 39 patients. J Pain Symptom Manage 2001; 22:834-842.

27. Fitzgibbon EJ, Hall P, Schroder C, Seely J, Viola R. Low dose Ketamine as an analgesic adjuvant in difficult pain syndromes: a strategy for conversion from parenteral to oral Ketamine. J Pain Symptom Manage 2002; 23:165-170.

28. Bell RF, Eccleston C, Kalso E. Ketamine as adjuvant to opioids for cancer pain. A qualitative systematic review. J Pain Symptom Manage 2003; 26:867-875.

29. Fisher K, Coderre TJ, Hagen NA. Targeting the $\mathrm{N}$-methyl-D-aspartate receptor for chronic pain management. Preclinical animal studies, recent clinical experience and future research directions. I Pain Symptom Manage 2000; 20:358373. 